\title{
A Study of Telemedical Applications Based on User Experience
}

\author{
Joanna Rybka ${ }^{1,2}$, Małgorzata Plechawska-Wójcik ${ }^{3}$, Tomasz Tokarek ${ }^{4}$
}

1 Department of Public Health, Nicolaus Copernicus University in Toruń, Poland

2 Research Center for Entrepreneurship and Innovation, Hasselt University, Belgium

3 Institute of Computer Science, Lublin University of Technology, Poland

4 Department of Pathomorphology, Student's Pathomorphology Group, Jagiellonian University in Kraków, Collegium Medicum, Poland

\begin{abstract}
The popularity of telemedical applications has been increasing noticeably in recent years. Easy access to a variety of software products makes contemporary users concentrate not only on functionalities but also on the design of the Graphical User Interface (GUI) - its usefulness, ease of use, and intuitiveness. The objective of this study was to evaluate the GUI quality of selected telemedical applications dedicated to depressed patients and doctors providing medical care for this group of patients. Another aim of the paper was to propose and check assessment criteria applied to the evaluation experiment. To achieve this objective, the case study was based on two methods: expert analysis and cognitive walkthrough. The expert analysis study was conducted on three groups of users: user experience specialists, patients, and clinicians. Although the expert analysis method is usually dedicated to GUI design specialists, in this study, we also applied it to patient and physician groups. The results showed that there were no statistical differences between assessments carried out by those three groups of users. Applied testing criteria were revised with Nielsen's heuristics. The proposed criteria helped to uncover many usability problems in several different areas during user tests. Further studies might be performed in order to strengthen accuracy and for completion of the proposed expert analysis evaluation criteria. However, our results show that the criteria we used seem to be robust enough to apply to both expert and end-user evaluations. Furthermore, multiple evaluation based on two different methods presented with better results, allowing not only problem identification but also verification.
\end{abstract}

\section{Introduction}

The market of telemedical applications has been growing rapidly. Medical applications supporting patients, clinicians, or both are becoming more and more popular. According to the definition (American Telemedicine As- 
sociation, 2012), telemedicine is "the use of medical information exchange from one site to another via electronic communications to improve patients' health status". Although first generation telemedicine tools were developed in the late 1960s, successful solutions began to appear in 1990s, when communication technologies became more available. Since then, the term "telemedicine" has evolved into telehealth and - afterwards - eHealth. According to S. Koch (2006), eHealth is an emerging field in the intersection of medical informatics, public health and business, referring to health services and information delivered or enhanced through the Internet and related technologies. In a broader sense, the term characterizes not only a technical development, but also a state-of-mind, a way of thinking, an attitude, and a commitment for networked, global thinking, to improve healthcare locally, regionally and worldwide by using information and communication technology.

Although scientific evidence of the effects of home telehealth solutions is still scarce (especially outside the USA), experts have observed trends of worldwide increase in the use of telemedicine and in the e-Health market. Reports (InMedica, 2012) show that in 2012, more than 30,000 patients around the world were monitored remotely by providers. Such support is first of all offered to patients suffering from heart failure, diabetes, hypertension, pulmonary disease, and depression. According to InMedica reports, the number of patients receiving any form of telecare is expected to reach 1.8 million in just 4 years, mostly in the form of primary care services (Hertz, 2015).

The diversity of telemedical and e-health applications represents the variety of needs of their end-users. There are different types of solutions on the market, including medical portals, web and mobile applications, and telemedicine computer systems, all of which are becoming more and more useful and accessible. Modern applications offer a wide range of functionalities, such as forum and discussion groups as well as e-medical consultations or distance monitoring of a patient's health condition. Some applications are highly specialized. They might support the physician in the diagnosis and treatment process as well as in searching for and adjustment of medicines. Numerous applications are dedicated to self-monitoring and self-observation of patients. Such services enable users to control their symptoms, check medication compliance, and monitor treatment progress. Users may also maintain electronic diaries and registries, collecting and monitoring patients' data between regular medical visits. Such applications are often based on dedicated equipment that is able to measure specified bodily functions, such as physiological and biochemical parameters. 
Among the most popular telemedical and e-health applications are interactive applications dedicated to both doctors and patients supporting doctor-patient interaction. What is more, such solutions offer a great opportunity for tightening the relationship between doctors and patients. Applications supporting communication between patient and doctor give patients access to cost-effective medical services supported by the use of real-time technologies (Video Conferencing Advice, 2015). Collecting real-life data is also a great opportunity for doctors and scientists, because these data are usually embedded in the context of patients' daily health habits, mood, and emotions. Furthermore, telemedical solutions support pre- and post-hospital management, which is crucial for medical outcome and often important for patient survival (Ackerman et al., 2012).

Currently, there are many different medical applications available on the market. A properly adjusted set of functionalities that ensures a positive user experience can determine whether the application will be successful and attract enough users. Graphical user interfaces allow users to interact with electronic devices through their graphical elements and visual indicators. It is extremely important to keep standards and conventions, while simultaneously attracting users with an interesting interface that will encourage them to share their medical and personal data. A welldesigned interface is an extremely important factor because it determines users' interest and attention. Applications need to be able to focus the attention on the user and simultaneously consider the capabilities and limitations of the used technology (Rubin et al., 2008). Mobile applications need to have interfaces adapted to user needs and recommendations. Personalization is also an important factor (Prenzel et al., 2012). Medical applications need to be adjusted to different people from diversified age groups, with different computer abilities and performance. What is more, rapid development of mobile devices (tablets and smartphones) results in the need to adapt applications to different resolutions and conditions (Resmini et al., 2011) to meet customer needs. Intuitive and functional interfaces constitute important requirements for modern web and mobile applications. Application functionalities need to be realized in a way that is comprehensible for users. User Experience (UX) helps to meet these requirements. Recent research (Isomursu et al., 2012) shows that the User Experience Design has become more and more industry relevant in recent years, and its role is still expanding. The significant impact of UX on web and mobile applications has been explored by numerous authors (Blomkvist, 2005; Chamberlain et al., 2006; Dayton et al., 2009; Detweiler, 2007; Dhir et al., 2013). 
In order to improve quality and usability of upcoming tools, evaluation of existing solutions may provide valuable insight for further development. The objective of this study was evaluation of graphical user interfaces of selected telemedical applications supporting communication between doctor and patient. Two popular and well-ranked medical applications were chosen to be tested. The study was based on applications dedicated to patients who suffer from depression and physicians who care for those patients. We decided to focus on this group of patients for several reasons. Patients suffering from depression represent a specific group of users who are characterized by cognitive impairments, changes in consciousness, and problems with concentration. Therefore, applications for depressed patients require a specially designed graphical user interface that takes the mental and cognitive limitations of these users into consideration. More specifically, such a GUI needs to support clear, undisturbed perception of the content and functionalities of the application. The layout should be easy to understand and navigate and, simultaneously, the GUI should avoid overwhelmingly distracting and irritating elements. Considering the increasing role of medical applications dedicated to mood disorders, it is important to develop tools which are well-received by users.

The aim of this study was to define evaluation criteria and validate methods which can be used for the assessment of graphical user interfaces in medical applications. To make the assessment complete, the case study covered several user groups: patients, clinicians, and ITC specialists experienced in GUI design. By including different levels of users, we aimed to achieve a more comprehensive analysis and increase the ability to validate proposed assessment criteria.

The structure of this paper is as follows. The next chapter provides details regarding the methods employed, providing descriptions of user experience analysis. The following chapter presents the results of the user experience analysis. A summarizing discussion is presented in the last chapter.

\section{Materials and Methods}

To ensure quality of medical applications, such applications must be checked and tested by different users working on different types of devices. In order to assess users' viewing experience, dedicated GUI quality evaluation methods may be used. There are several requirements for the GUI: it should be ergonomic, intuitive, and easy and fast in use. Applications should also be adjusted to different screen resolutions (computer monitors, smart phones, 
tablets, etc). The GUI should be readable with a minimum of resizing, panning, and scrolling.

GUI quality can be assessed using expert analysis. Expert analysis is one of the most widely used methods in application testing. Experts, while using the application, check the predefined areas and mark inaccuracies on a special form. Areas are defined to help to examine application compliance with interface design guidelines, such as Nielsen-Molich heuristics (Nielsen et al., 1990), and to detect potential problems. Predefined areas are usually divided into several detailed sub-areas. What is more, sub-areas usually have assigned detailed questions to help experts cover all important aspects of GUI quality (Laskowski, 2012). Expert analysis is also considered to be one of the most reliable methods. It has been shown (Krug, 2000) that in most cases, a group of several experts using adequate methodology is able to detect and correct over $85 \%$ of errors in a given software. In particular, this applies to errors and inaccuracies regarding GUI elements. It should be noted that testing can never completely identify all the defects within software (Pan, 1999). However, it is very important to have an assessment performed by non-experts, who are usually regular users of the application tested (also referred to as target user group or end-users). User opinions reveal whether the application is comprehensible and what areas might be difficult to navigate (Mathis, 2011). A typical usability test is divided into planning, execution, analysis, and report preparation.

In order to ensure the effectiveness of the GUI evaluation, it is necessary to define the most important tasks for users (Allen et al., 2012). In this study, we decided to obtain evaluations from end-users, including patients and clinicians, as well as from the experts. Although end-users' experience is the most important for the success of an application, experts know user interface design fundamentals and principles, so they can clearly indicate errors and give advice and tips concerning GUI elements. Therefore, having both users and experts in our study allowed a more comprehensive assessment to be made. Furthermore, all groups assessed the applications using the same criteria, which consisted of a detailed list of questions assigned to specific areas and subareas. With this approach, we could compare the answers from patients, clinicians, and experts and look for commonalities and differences in their assessments. The list of criteria (Table 1) was an authorial set of questions based on the heuristics and good practices applied in GUI design and quality improvement. Table 2 presents the grading scale used to assess each evaluated area. According to some studies (Shneiderman, 1998), user experience of the same GUI changes over time. For instance, novice users interacting with a system for the first time prefer simple actions, whereby 
Table 1. The list of predefined testing areas with questions assigned

\begin{tabular}{|c|c|}
\hline \multicolumn{2}{|l|}{ Application interface } \\
\hline \multirow{3}{*}{ Layout } & Is the application readable? \\
\hline & Is the application displayed properly on your screen? \\
\hline & Are interface elements arranged properly? \\
\hline \multirow{2}{*}{ Color scheme } & Is there proper contrast between text and background? \\
\hline & Is the color scheme readable? \\
\hline \multicolumn{2}{|c|}{ Navigation and data structure } \\
\hline \multirow{2}{*}{ Ease of use } & Is the access to all sections and functions of the application easy? \\
\hline & Is it easy to get around the application? \\
\hline \multirow{2}{*}{$\begin{array}{l}\text { Information hierarchy } \\
\text { and structure }\end{array}$} & Is the menu easy to use? \\
\hline & Is it easy to find required information? \\
\hline \multicolumn{2}{|c|}{ Feedback, system messages, user help } \\
\hline $\begin{array}{l}\text { System messages } \\
\text { (general) }\end{array}$ & Does the application respond properly to your actions? \\
\hline \multirow{2}{*}{$\begin{array}{l}\text { System messages } \\
\text { (errors) }\end{array}$} & $\begin{array}{l}\text { Does the application give a hint when there is a problem using } \\
\text { the application? }\end{array}$ \\
\hline & $\begin{array}{l}\text { Is it easy to perform actions suggested by the application in order } \\
\text { to solve problems with using the application? }\end{array}$ \\
\hline \multicolumn{2}{|l|}{ Content } \\
\hline Naming & Are labels and headlines easy to understand? \\
\hline Page text & Is the application content easy to understand? \\
\hline \multicolumn{2}{|l|}{ Data input } \\
\hline \multirow{3}{*}{ Data } & When you provide data, does the application check it for mistakes? \\
\hline & $\begin{array}{l}\text { Does the application guide you regarding how to provide the data } \\
\text { (e.g. on format or data range)? }\end{array}$ \\
\hline & Is it easy to fill-out the form? \\
\hline
\end{tabular}

fast and easy learning is of great importance (Phung, 2007). In order to avoid any influence from previous experience, the assessments were made by users who had never used the applications before. The same rule was applied for the experts that were included in the study.

Cognitive walkthrough was also applied during the study. It is a popular usability and GUI quality testing method. It enables one to check the ease of interface learning during initial contact with the system (Wharton et al., 1994). The method requires a few tasks to be performed by a user as they work with an application. The goal is to check the flow of the pro- 
Table 2. The grading scale

\begin{tabular}{|c|l|}
\hline Grade & \multicolumn{1}{|c|}{ Description } \\
\hline 1 & $\begin{array}{l}\text { Critical usability errors were observed, preventing normal usage or discouraging } \\
\text { the user from using the application. }\end{array}$ \\
\hline 2 & $\begin{array}{l}\text { Serious usability issues were encountered, which may prevent most users from } \\
\text { task realization. }\end{array}$ \\
\hline 3 & $\begin{array}{l}\text { Minor usability issues were observed, which, if accumulated, may have a negative } \\
\text { impact on user performance. }\end{array}$ \\
\hline 4 & $\begin{array}{l}\text { Single minor usability issues were observed, which may have a negative impact } \\
\text { on user work quality (e.g. poor readability). }\end{array}$ \\
\hline 5 & $\begin{array}{l}\text { No usability issues influencing either user performance or work quality were } \\
\text { identified. }\end{array}$ \\
\hline
\end{tabular}

cesses undertaken by the user. This relatively low-cost and quick method is suitable for use at different stages of the design process, including the final version of the application. Research shows (Koyani et al., 2004) that cognitive walkthrough is also efficient in the process of detecting potential interface problems. In particular, it checks the following aspects (PlechawskaWójcik, 2014):

1. Does the user know what to do during the analyzed step?

2. If the action performed by the user is correct, are they aware of it?

3. If the action performed by the user is correct, does he or she feel as if they are getting closer to reaching the goal?

\section{Results}

In our case study, we tested two well-known telemedical applications designed to overcome depression. The applications chosen were: This Way Up and RealTime Health. This Way Up is a typical self-observation and diagnosis application supporting remote monitoring of patients' health. This application has separate versions dedicated to patients and to clinicians. A web application for patients supports them in adhering to treatment, self-testing of symptoms, and disease monitoring. Patients also receive support from their clinician through this application. In addition, patients can take selfpaced courses, including skill building courses. This Way Up for clinicians is a web application which supports physicians in monitoring patients remotely, supporting patients in treatment, and measuring clinical outcomes. The application also provides medical information, technical training, and help to develop treatment strategies. 
RealTime Health is a mobile application also dedicated to people suffering from depression. The application enables users to communicate with other patients and share their experiences in the form of patient storytelling, video stories, and evidence-based practice. Patients may also get answers to health-related questions. Through sharing first-hand stories, the application complements medical knowledge and improves understanding of depression as a disease. The application also motivates patients to proactively selfmanage their health and learn about the day-to-day challenges of living with depression.

These three applications (RealTime Health and two separate versions of the This Way Up application) were tested by three groups of test users: a target patient group, a target clinician group, and a UX (User experience) specialist group. All together, forty-five respondents took part in the experiment. The target patient group consisted of fifteen people and the target clinician group contained twenty persons. Ten respondents took part in the UX (User experience) specialist test. All presented and discussed numerical results with regard to the mean values obtained separately for each question and for each testing group. All results were gathered using Google Form and Google Spreadsheets.

The scoring criteria presented in Table 2 were applied. Fifteen users representing the target user group were selected to test both applications (This Way Up and RealTime Health) for patients. The results are presented in Table 3.

The results allow one to conclude that the overall assessment of both interfaces was positive - respectively 4.43 for This Way Up and 4.375 for RealTime Health. The interface of This Way Up achieved a slightly higher score compared with RealTime Health. However, the differences were not statistically significant.

Testers indicated that the readability of the This Way Up interface needed improvement in the area of contrast and color. In this area, the application was rated 4 , whereas the average overall assessment was 4.43 . The same area in the RealTime Health application was assessed to be slightly better (4.25 points; the overall assessment: 4.375 ).

Moreover, the feedback section shows that both applications did not provide sufficient hints to help users who had problems with the application. Nevertheless, users found navigation to be efficient and well-planned. RealTime Health scored a little bit worse than This Way Up in such areas as navigation and data structure and feedback, system messages, user help, and data input. 
Table 3. Results of expert analysis

\begin{tabular}{|c|c|c|c|c|c|}
\hline \multirow[b]{2}{*}{ Questions } & \multicolumn{2}{|c|}{ Patients } & \multirow{2}{*}{$\begin{array}{c}\text { Clinicians } \\
\text { Uhis Way }\end{array}$} & \multicolumn{2}{|c|}{ UX specialists } \\
\hline & $\begin{array}{l}\text { This Way } \\
\text { Up }\end{array}$ & $\begin{array}{r}\text { RealTime } \\
\text { Health }\end{array}$ & & $\begin{array}{l}\text { This Way } \\
\text { Up for } \\
\text { Patients }\end{array}$ & $\begin{array}{l}\text { This Way } \\
\text { Up for } \\
\text { Clinician }\end{array}$ \\
\hline Application interface & 4.375 & 4.5 & 4.549 & 4.48 & 4.76 \\
\hline Is the application readable? & 4.625 & 4.625 & 4.615 & 3.8 & 4.6 \\
\hline $\begin{array}{l}\text { Is the application displayed prop- } \\
\text { erly on your screen? }\end{array}$ & 4.75 & 4.625 & 4.69 & 4.8 & 4.8 \\
\hline $\begin{array}{l}\text { Are interface elements arranged } \\
\text { properly? }\end{array}$ & 4.625 & 4.625 & 4.67 & 4.2 & 4.4 \\
\hline $\begin{array}{l}\text { Is there proper contrast between } \\
\text { text and background? }\end{array}$ & 4.0 & 4.25 & 4.31 & 4.8 & 5.0 \\
\hline Is the color scheme readable? & 3.875 & 4.375 & 4.46 & 4.8 & 5.0 \\
\hline Navigation and data structure & 4.53 & 4.31 & 4.30 & 3.85 & 4.05 \\
\hline $\begin{array}{l}\text { Is access to all sections and func- } \\
\text { tions of the application easy? }\end{array}$ & 4.625 & 4.375 & 4.285 & 3.8 & 4.2 \\
\hline $\begin{array}{l}\text { Is it easy to navigate within the } \\
\text { application? }\end{array}$ & 4.375 & 4.125 & 4.285 & 4.0 & 4.2 \\
\hline Is the menu easy to use? & 4.75 & 4.375 & 4.57 & 4.0 & 3.6 \\
\hline $\begin{array}{l}\text { Is it easy to find required informa- } \\
\text { tion? }\end{array}$ & 4.375 & 4.375 & 4.07 & 3.6 & 4.2 \\
\hline $\begin{array}{l}\text { Feedback, system messages, user } \\
\text { help }\end{array}$ & 4.29 & 4.21 & 4.26 & 4.13 & 4.33 \\
\hline $\begin{array}{l}\text { Does the application respond } \\
\text { properly to your actions? }\end{array}$ & 4.5 & 4.375 & 4.43 & 4.6 & 4.8 \\
\hline $\begin{array}{l}\text { Does the application give a hint } \\
\text { when there is a problem with us- } \\
\text { ing the application? }\end{array}$ & 4.125 & 4.125 & 4.07 & 3.4 & 4.0 \\
\hline $\begin{array}{l}\text { Is it easy to perform actions sug- } \\
\text { gested by the application in order } \\
\text { to solve problems with using the } \\
\text { application? }\end{array}$ & 4.25 & 4.125 & 4.285 & 4.4 & 4.2 \\
\hline Content & 4.56 & 4.5 & 4.475 & 4.7 & 4.4 \\
\hline $\begin{array}{l}\text { Are labels and headlines easy to } \\
\text { understand? }\end{array}$ & 4.5 & 4.5 & 4.64 & 4.8 & 4.4 \\
\hline $\begin{array}{l}\text { Is the application content easy to } \\
\text { understand? }\end{array}$ & 4.625 & 4.5 & 4.31 & 4.6 & 4.4 \\
\hline Data input & 4.46 & 4.33 & 4.19 & 4.13 & 4.13 \\
\hline $\begin{array}{l}\text { When you provide the data, does } \\
\text { the application check if there are } \\
\text { any mistakes? }\end{array}$ & 4.5 & 4.375 & 4.43 & 4.0 & 4.0 \\
\hline $\begin{array}{l}\text { Does the application guide you } \\
\text { regarding how to provide the data } \\
\text { (e.g. on format or data range)? }\end{array}$ & 4.5 & 4.25 & 4.07 & 4.0 & 4.0 \\
\hline Is it easy to fill-out the form? & 4.375 & 4.375 & 4.07 & 4.4 & 4.4 \\
\hline The entire interface & 4.43 & 4.375 & 4.37 & 4.235 & 4.36 \\
\hline
\end{tabular}


The This Way Up application dedicated for clinicians was also wellassessed by the target group of fifteen clinicians. The version of This Way Up dedicated for clinicians was rated a little bit worse than This Way Up for patients. However, this difference was not statistically significant. According to the users, the registration form, which was found to be complicated and not supported with a tutorial or manual, was the main obstacle in using This Way Up. Users were concerned about fonts; however, the worst rated section of the application was Navigation and data structure. Nevertheless, users reported only minor errors in the section entitled Application interface.

Assessment of target users was compared with the results of a survey completed by a group of ten UX specialists. UX specialists assessed the application This Way Up (both versions - for patients and clinicians). According to the results presented in Table 3, the assessment of UX specialists agrees with the results achieved by target users. There is no statistical difference between results of end-users and UX specialists. According to respondents, the most important problem with the interfaces of both versions of the This Way Up application is lack of validation messages. There are also identified problems with navigation, especially in the application dedicated to patients. It is easy to lose orientation in the application; there are also problems with returning to the main page.

The obtained results prove that the interfaces of the chosen medical applications are well-designed. Both patients and clinicians evaluated the tested applications well. What is more, UX professionals confirmed those results. The method helped to detect specific and minor problems, whereas assessments performed by different groups provided complementary information.

Both versions of the application This Way Up (dedicated to patients and clinicians) were also analyzed using the cognitive walkthrough method. For the purpose of this analysis, a set of user tasks was proposed. Tasks were diversified to cover main aspects of the application, including data forms, navigation, and menu structure.

This Way Up - tasks for patients

1. Register as a patient using arbitrary data.

2. Enroll in a Depression course.

3. Run the diagnosis program for Depression.

4. Find the Activity Monitoring and Planning resource for the Depression course.

This Way Up - tasks for clinicians

1. Prescribe a THIS WAY UP Clinical Depression course for one patient. 
2. Find the course prescription list and check details (code, name of the patient) of the first position.

3. Find example homework for a patient with depression.

4. Find the Pan Expiry Dates for all of your currently valid prescriptions.

The cognitive walkthrough study was carried out by five test users. All tasks were accomplished. However, permanent performance problems occurred during completion of several tasks. Such problems were encountered during clinician account managing. The second issue highlighted by all respondents was unintuitive navigation in the Pan Expiry Dates section (task no. 4). Users found it problematic to accomplish this task due to labels not being clearly named.

Tasks in the patient section were also accomplished successfully, except for task no. 3: Run diagnosis program for Depression. It was impossible to finish the lesson due to performance problems (slideshow suspended). Communication between patient and clinician involving invitation management was successful. Navigation in the application was intuitive. Users found it easy to navigate through the application and to run particular functionalities. Messages, data structure, and system messages were also well evaluated. Clearly laid questions and a help window presenting available options displayed at the beginning of the course contributed to fast and smooth completion of tasks. However, the application ran slowly and users were concerned when they needed to wait for a page to process without any message regarding what caused the delay. This caused users to feel a lack of control over the application.

\section{Discussion}

The huge impact of interface quality on the way users choose and work with applications is more and more perceivable (Resmini et al., 2011), while end-users have become conscious consumers who make informed choices. Most of them make their choice based on the interface design. What is more, even if they are not are aware of it, the huge role user experience plays in an application's success has been scientifically proven. Easy access to a variety of software products makes contemporary users concentrate not only on functionalities but also on the design of the GUI - its usefulness, ease of use, and intuitiveness. It is of great importance for software developers to develop graphical user interfaces meeting users' expectations. There are numerous methods and rules for both designing and testing GUI quality 
and user experience. Diversification of methods is caused by the necessity of adjusting them to the type of test users and the developmental phase of the tested application.

The objective of this study was to evaluate the GUI quality of selected telemedical applications dedicated to supporting patients with depression and the doctors who treat them. The aim of the paper was also to propose and check assessment criteria applied to the evaluation experiment. Wellknown telemedical applications were chosen as example applications to be verified in the study. This Way Up for patients and clinicians and Real Time Health are telemedical applications dedicated to those who struggle with depression. People suffering from this disease represent a group of particularly sensitive users who should work with a dedicated, well-designed, usable interface. The applications analyzed in the case study were dedicated to helping tackle the same disease; however, they each had a different set of functionalities.

To achieve the objective of the paper, the case study was based on two methods: expert analysis and cognitive walkthrough. The expert analysis study was conducted on three different groups of users: UX (User experience) specialists, patients, and clinicians. Test users where chosen among three different groups: dedicated target users - patients and clinicians, and UX experts. Their responses were gathered separately and compared in the study. Criteria proposed for the expert analysis were adjusted for those groups. Users, while testing applications, filled-out a specially prepared usability questionnaire. The questionnaires covered the most important aspects of the interface design. They were divided into the following sections: Application interface, Navigation and data structure, Feedback, system messages, user help, Content, and Data input. Both interfaces were rated positively. A detailed analysis showed no statistical differences between responses regarding tested applications, although there were some issues identified. Although the expert analysis method is usually dedicated to GUI design specialists, in this study we applied it also to the patient and physician groups. The results show that there are no statistical differences between assessments carried out by those three user groups. This proves that in the case of dedicated, specialized software, well- matched end-users might occur as valuable test users as experts. It shows that in the case of expert analysis, similar to cognitive walkthrough, although it is recommended that evaluators typically be experts, such as usability specialists, the method can be applied successfully in many different situations with the participation of general IT specialists or dedicated users. What is more, in contrast to popular claims, experts usually do not guarantee better results 
in completing tasks or answering interface-related surveys. This is especially true if neither they nor typical end-users have seen the tested application before the experiment. Their expert status, experience, and knowledge allow them, however, to perform testing tasks faster than the novices and to spend less time on them (Dillon et al., 1997; Jochen et al., 1991).

Expert analysis is often used in conjunction with other GUI quality tests to supplement results and identify potential problems. In this study, results of expert analysis were confirmed by cognitive walkthrough analysis. The cognitive walkthrough was performed by UX experts, who solved tasks defined separately for applications dedicated to clinicians and patients. Although most of the tasks were completed successfully, some of them occurred to be problematic. The study identified performance problems as well as unintuitive navigation and poor feedback issues. In general, results of the cognitive walkthrough confirmed results obtained in the expert analysis study and further supported our approach to evaluation of tested applications. Based on these results, the criteria that we proposed might be regarded as relevant for use with different user groups and different applications developed using different technologies. Studies show (Krug, 2000) that in most cases, a well-matched test group using adequate methodology is able to detect over $85 \%$ of errors in software, including errors in GUI quality. This method, in combination with cognitive walkthrough, seems to further increase the reliability of GUI quality assessment.

The testing criteria that we applied in this case study were also revised with heuristics proposed by Nielsen et al. (1990). Using heuristics implied that an expert reviewed an interface against a defined set of guidelines. These heuristics provide a template to help uncover problems likely encountered by users. Nielsen's 10 heuristics are the most popular, but there are actually many more.

In this study, expert analysis and cognitive walkthrough were principal methods and heuristics were used to validate the case study assumptions and criteria, whereas typical heuristic evaluation was not performed. The heuristics referred to are 10 general principles for interaction design. They are available on the Internet (Nielsen et al., 1990; Sauro, 2011) and include:

1. Visibility of system status - The application should always keep users informed about actions actually performed through appropriate feedback.

2. Match between system and real world - The phrases and terms used in the application should be clear for users. System-oriented terms should be avoided. 
3. User control and freedom - The application should support users' undo and redo actions done by mistake.

4. Consistency and standards - The convention applied in the application should be consistent; the same terms and phrases used in different places should have the same meaning.

5. Error prevention - Well-designed error messages are important, but even more important is preventing a problem from occurring by eliminating error-prone conditions.

6. Recognition rather than recall - Instructions should be visible and easily retrievable; users should not be obliged to remember information from different places in the application.

7. Flexibility and efficiency of use - Users familiarized with the application should be able to take advantage of accelerators supporting performance of frequent actions.

8. Aesthetic and minimalist design - Irrelevant or rarely needed information should be hidden to not distract the user.

9. Help for users, allowing them to recognize, diagnose, and recover from errors - If an error occurs, an appropriate message should be displayed to suggest a solution.

10. Help and documentation - It is better if the application is comprehensible without documentation, but if it is necessary, it should be easy to find.

Criteria proposed in our case study covered the heuristics in the following way. The Application interface section covered the $4^{\text {th }}$ and $8^{\text {th }}$ heuristics through questions about proper element arrangement and readability. The Navigation and Data Structure section goes with the $4^{\text {th }}, 6^{\text {th }}$, and $10^{\text {th }}$, since it contained questions about accessing required information, ease of use, and division into sections. Feedback, system messages, and user help covered heuristics such as the $1^{\text {st }}, 5^{\text {th }}, 9^{\text {th }}$ and $10^{\text {th }}$, including specific questions regarding the application's responses and suggestions. The Content section, containing questions about clarity of phrases, corresponded to the $2^{\text {nd }}$ and $8^{\text {th }}$ heuristics. The question about errors placed in the Data Input section covered the $3^{\text {rd }}$ heuristic. Because the study was dedicated to novice users, the $7^{\text {th }}$ heuristic, which is dedicated to users familiarized with the application, was omitted.

The criteria presented in this study helped to uncover many usability problems in several different areas during user tests. Further studies might be performed in order to strengthen accuracy and complete the expert analysis evaluation criteria proposed in this paper. However, our results show that the criteria we proposed seem to be robust enough to apply in both 
expert and end-user evaluations. Furthermore, multiple evaluation based on two different methods presented with better results, allowing not only identification but also verification of problems.

\section{R E F E R E N C E S}

Ackerman, M., Craft, R., Ferrante, F., Kratz, M., Mandil, S., \& Sapci, H. (2012). Telemedicine technology. Telemedicine Journal and e-Health, 8(1), 71-78.

Allen, J., \& Chudley, J. (2012). Smashing UX Design: Foundations for Designing Online User Experiences. Chichester, West Sussex, U.K.: John Wiley \& Sons Ltd.

American Telemedicine Association. (2013). What is Telemedicine? Retrieved from http://www.americantelemed.org/about-telemedicine/what-is-telemedicine \#.VBafOfl_t8E

Blomkvist, S. (2005). Towards a Model for Bridging Agile Development and UserCentered Design. Human-Centered Software Engineering - Integrating Usability in the Software Development Lifecycle. Human-Computer Interaction Series, 8(IV), 219-244.

Chamberlain, S., Sharp, H., \& Maiden, N. (2006). Towards a Framework for Integrating Agile Development and User-Centered Design. Extreme Programming and Agile Processes in Software Engineering. Lecture Notes in Computer Science, 4044, 143-153.

Dayton, D., \& Barnum, C. (2009). The Impact of Agile on User-centered Design: Two Surveys Tell the Story. Technical Communication, 56(3), 219-234.

Detweiler, M. (2007). Managing UCD Within Agile Projects. Interactions, 14(3), $40-42$.

Dhir, A., \& Al-Kahtani, M. (2013). A Case Study on User Experience (UX) Evaluation of Mobile Augmented Reality Prototypes. Journal of Universal Computer Science, 19(8), 1175-1196.

Dillon, A., \& Song, M. (1997). An empirical comparison of the usability for novice and expert searchers of a textual and a graphic interface to an art-resource database. Journal of Digital Information, 1(1), 619-638.

Hertz, B. T. (2015). Telemedicine - patient demand, cost containment drive growth. Retrieved from http://medicaleconomics.modernmedicine.com/medical-econ omics/news/modernmedicine/modern-medicine-feature-articles/telemedicin e-patient-demand-c

InMedica Report. (2012). The World Market for Telehealth - An Analysis of Demand Dynamics. Retrieved from http://www.e-health-com.eu/fileadmin/use r_upload/dateien/Downloads/Telehealth_-_An_Analysis_of_Demand_Dynam ics_-_World_-_2012_-_Brochure.pdf

Isomursu, M., Sirotkin, A., Voltti, P., \& Halonen, M. (2012). User Experience Design Goes Agile in Lean Transformation - A Case Study. Agile Conference (AGILE), IEEE, 1-10. 
Jochen, P. (1991). Errors in computerized office work: Difference between novice and expert users. ACM SIGCHI Bulletin, 23(2), 63-66.

Koch, S. (2006). Home telehealth - current state and future trends. International Journal of Medical Informatics, 75(8), 565-576.

Koyani, S. J., Bailey, R., \& Nall, J. R. (2004). Research-Based Web Design \& Usability Guidelines. Washington, DC, U. S.: National Institutes of Health.

Krug, S. (2000). Don't Make Me Think! A Common Sense Approach to Web Usability. New York: Que.

Laskowski, M. (2012). Proposals for application interface testing methods. Informatyka Automatyka Pomiary w Gospodarce i Ochronie Środowiska, 4b, 21-24 (in Polish).

Mathis, L. (2011). Designed for Use. Dallas: The Pragmatic Bookshelft.

Nielsen, J., \& Molich, R. (1990). Heuristic evaluation of user interfaces. In Proceedings of the SIGCHI conference on Human factors in computing systems: Empowering people, ACM, New York, USA, 249-256.

Pan, J. (1999). Software Testing. Retrieved from http://www.ece.cmu.edu/ ${ }^{\sim}$ koopm an/des_s99/sw_testing

Phung, Q. (2007). Identifying Usability Problems in relation to User Experience with System and Tests. Retrieved from http://projekter.aau.dk/projekter/fil es/61070563/1181058253.pdf

Plechawska-Wójcik, M. (2014). Heuristic evaluation and user experience assessment of online shopping portals using cognitive walkthrough and expert method. MakeLearn 2014: Human Capital without Borders; Knowledge and Learning for Quality of Life. Proceedings of the Management, Knowledge and Learning International Conference (pp. 467-475).

Prenzel, A., \& Ringwelski, G. (2012). Design of Human-computer Interfaces in Scheduling Applications. Proceedings of ICEIS Conference, 219-228.

Resmini, A., \& Rosati, L. (2011). Pervasive Information Architecture: Designing Cross-Channel User Experiences. Morgan Kaufmann Publishers.

Rubin, J., \& Chisnell, D. (2008). Handbook of Usability Testing, Second Edition: How to Plan, Design, and Conduct Effective Tests. Indianapolis: Wiley Publishing Inc.

Sauro, J. (2011). What's The Difference Between A Heuristic Evaluation And A Cognitive Walkthrough? Measuring Usability. Retrieved from http://www. measuringusability.com/blog/he-cw.php

Shneiderman, B. (1998). Designing the user interface. Boston, MA, USA: AddisonWesley Longman.

Video Conferencing Advice. (2015). Overview of Major Telemedicine Solutions. Retrieved from http://www.videoconferencingadvice.com/telemedicine-solutio ns.html

Wharton, C., Rieman, J., Lewis, C., \& Polson, P. (1994). The cognitive walkthrough method: A practitioner's guide. In J. Nielsen, \& R. L. Mack (Eds.). Usability inspection methods. New York, NY: John Wiley \& Sons Inc. 